\title{
Tartaric Acid New Synthetic Derivatives Antibacterial Activity against the Phytopathogenic Pseudomonas syringae
}

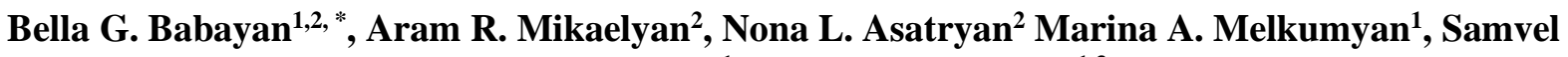 \\ A. Bagdasaryan ${ }^{1}$, Anna M. Grigoryan ${ }^{1,3}$ \\ ${ }^{1}$ National Polytechnic University of Armenia (NPUA), Republic of Armenia (RA) \\ 2"Armbiotechnology" Scientific And Production Center (SPC), National Academy of Sciences (NAS), Republic \\ of Armenia (RA) \\ ${ }^{3}$ Russian-Armenian (Slavonic) University \\ *Corresponding author
}

\begin{abstract}
.
Antimicrobial resistance is one of the most pressing issues of our time, according to WHO's reports over the past several years. And day by day the further aggravation of the mentioned problem is being caused by increase in antibiotic usage values in various scopes of mankind activity. That is why, the search of novel ecologically safe alternatives of classical antibiotic therapy against pathogen microorganisms is very significant direction of research world around. And it is important not only in case of human pathogens, but also for opportunistic pathogenic microorganisms, pathogens of plants and animals that have a significant impact on public health and agriculture.

During this research, 4 new synthetic derivatives of natural antimicrobial compound tartaric acid, were elaborated at the laboratory of new agrarian pesticides creation and the quality control of National Polytechnic University of Armenia (NPUA). Their biological activity and biodegradation potential were tested on different soil microorganisms, such as like various strains of phytopathogenic Pseudomonas syringae, from The National Collection of Microorganisms of Microbe Depository Centre (MDC), "Armbiotechnology" Scientific \& Production Center (SPC), National Academy of Sciences (NAS), Republic of Armenia (RA).

According to results, complex salts of tartaric acid are effective as the prospective and a comparably ecologically safe alternative for combating antimicrobial resistance in this plant pathogen $P$. syringae. Besides, the obtained substances are effective against the other opportunistic pathogenic representatives of same genera, such as like $P$. aeruginosa and $P$. fluorescens. Moreover, the mentioned compounds are biodegradable by soil non-pathogenic $P$. chlororaphis.
\end{abstract}

Keywords: tartaric acid derivatives, Pseudomonas syringae, antimicrobial resistance, phytopathogen. 


\section{$2^{\text {nd }}$ International Conference on Advanced Research in SCIENCE, ENGINEERING \& TECHNOLOGY}

26-28 March, 2021

FRANCE,PARIS

\section{Introduction}

Tartaric acid is a natural aldaric acids representative, which is mostly presented in plants from algae to trees, being involved in various biosynthetic processes of their cells (Nunes et al., 2012; Cholet et al., 2016). This acid and the salts of it (tartrates) are well-known as antimicrobial active compounds, which are broadly used in chemical and food industry, as safe preservatives and conservation agents (DeBolt et al., 2006; Maroun et al., 2017).

One of the prospective directions of antimicrobial active compound elaboration is the derivatization of natural substances, such as like organic aldaric acid to the appropriate imides and amides (Khan, 2012; Malik, 2013).

Pseudomonas are well-known as Gram-negative bacteria with extremely high level of adaptation properties, including the resistance to various antimicrobial agents, such as like antibiotics. Beside they are capable of degradation of different natural and toxic synthetic xenobiotics. All these properties become a cause of additional complications for the treatment of pseudomonas infections in animal, human and plant organism (Chakhtoura, 2018; Adesoji et al., 2015).

$P$. syringae is the one of common phytopathogenic bacteria, represented world around. As a plant pathogen, it can infect a wide range of species, and exists as over 50 different pathovars. $P$. syringae overwinters on infected plant tissues such as regions of necrosis or gummosis (sap oozing from wounds on the tree) but can also overwinter in healthy looking plant tissues. In the spring, water from rain or other sources will wash the bacteria onto leaves/blossoms where it will grow and survive throughout the summer. Infection and pathogenesis of it by $P$. syringae tends to be favoured by wet, cool conditions - optimum temperatures for disease tend to be around $12-25^{\circ} \mathrm{C}$, although this can vary according to the pathovar involved. The bacteria tend to be seed-borne, and are dispersed between plants by rain splash. The mechanisms of $P$. syringae pathogenicity can be separated into several categories: ability to invade a plant, ability to overcome host resistance, biofilm formation, and production of proteins with ice-nucleating properties (Scholz-Schroeder et al., 2003; Jeong et al, 2016; Gomila et al., 2018).

These bacteria have a wide range of genetic mechanisms for synthesis of specific enzymes and that is why it has high level of antibiotic resistance, as well as high virulence with complex influence on plant immune system.

According to World Health Organization (WHO) reports, the problem of drug resistance and the spread of it among the various pathogens today is being extremely important. Thus, multidrug resistance is being considered as one of the research priorities of WHO for this century. It is driven by the overuse of antimicrobials in people, but also in animals, especially those used for food production, as well as in the environment. WHO is working with these sectors to implement a global action plan to tackle antimicrobial resistance by increasing awareness and knowledge, reducing infection, and encouraging prudent use of antimicrobials (Martin, 2011; Sundin and Wang, 2018; De Araujo et al., 2019).

In current paper there were researched the influence of 4 new synthetic derivatives of tartaric acid: benzylimide, cyclohexylimide, benzyl mono amino salt and cyclohexyl mono amino salt of tartaric acid on growth of some phytopathogenic strains of soil Pseudomonas syringae. 


\section{$2^{\text {nd }}$ International Conference on Advanced Research in SCIENCE, ENGINEERING \& TECHNOLOGY \\ 26-28 March, 2021 \\ FRANCE,PARIS}

\section{Methods}

The new synthetic derivatives of tartaric acid: 2 imides and 2 complex salts, which are more hydrophilic, were synthesized by two stage simple technology from the natural cream of tartar which was elaborated in laboratory of new agrarian pesticide creation and the quality control, NPUA (Dashchyan et al, 2014).

During the research there were used various non-pathogenic and opportunistic pathogenic strains of Pseudomonas syringae, P. aeruginosa, E. coli from the National Culture Collection of Microorganisms Depository Center at "Armbiotechnology" Scientific and Production Center of National Academy of Sciences of Republic of Armenia.

All the researched strains of microorganisms were cultivated on various liquid and solid nutrient agarised and selective cultural media. The selective media were containing 13 types of antibiotics of different classes and generations, mainly used in medicine, veterinary and agriculture, according to standard protocols. There selective cultural media were containing 50mkg/ml compatible antibiotic (Birger, 1982; Babayan, 2019; Babayan et al., 2019)

The following antibiotics of different classes and different generations were used: from $\beta$ lactamic - Pen/Penicillin, Amp/ampicillin, Amx/Amoxicillin, Amc/Augmentin, Cfx/Cefixime and Cro/Ceftriaxone; from aminoglycosides - Gen/Gentamicin, Kan/kanamycin, Str/Streptomycin; from fluoroquinolones - Cip/Ciprofloxacin; from tetracyclines Tcn/Tetracycline; from azalides of macrolides - Azm/azithromycin; from amphenicoles $\mathrm{Chl} /$ Chloramphenicol. All the used antibiotics were produced by "Astoria". As the positive and the negative control strains there were used the following antibiotic resistant and sensitive


DH5 $\alpha$ PEC7, as well as $P$. aeruginosa 9056 and P. aeruginosa 5249 strains.

The antimicrobial activity tests were done according to standard protocols (Babayan et al., 2019, Chaudhary et al., 2019).

\section{Results and Discussion}

During the tests of antibiotic resistance using 13 compounds, it was found out that the researched strains are sensitive only to: ampicillin, ceftriaxone, kanamycin, ciprofloxacin, and tetracycline.

The results of antimicrobial activity tests of 4 new synthetic derivatives of tartaric acid (Fig. 1) are represented on table 1.

Figure 1: Synthetic derivatives of tartaric acid: $a$-benzylimide of tartaric acid, $b$-cyclohexylimide of tartaric acid, $c$-benzyl mono amino salt of tartaric acid, $d$-cyclohexyl mono amino salt of tartaric acid.

$\mathbf{a}$

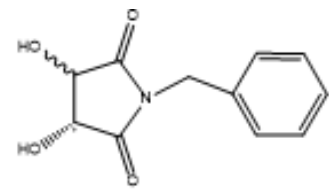

b

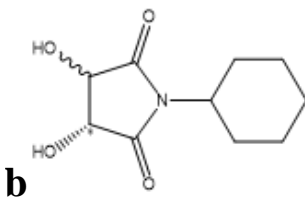

c

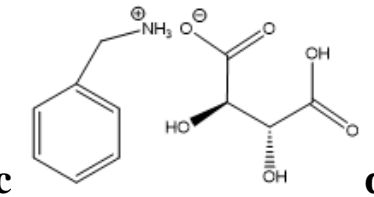

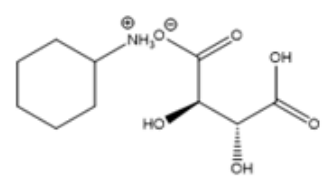

Source: researched in this paper. 


\section{$2^{\text {nd }}$ International Conference on Advanced Research in SCIENCE, ENGINEERING \& TECHNOLOGY \\ 26-28 March, 2021 \\ FRANCE,PARIS}

Table 1: The effect of TA derivatives on Pseudomonas syringae. The strains were cultivated on media with $0.5 \mathrm{M}$ and 0,1M consistence of TA derivatives: BI - benzylimide of TA, CI - Cyclohexylimide of tartaric acid, BAS mono amino salt of benzyl amine of tartaric acid; CAS-mono amino salts of cyclohexyl amine of TA; KT-tartrate of potassium, NKT - sodium potassium tartrate, TA - tartaric acid; “+”-growth; L-lysis of cells; the diameters of cells growth inhibition spots are presented in millimetres, $C$-control on nutrient agarised cultural media.

\begin{tabular}{|c|c|c|c|c|c|c|c|c|c|c|c|c|}
\hline \multirow{2}{*}{$\begin{array}{c}\text { Strain of } \\
\text { P. syringae }\end{array}$} & \multicolumn{2}{|c|}{ BAS } & \multicolumn{2}{|c|}{ BI } & \multicolumn{2}{c|}{ CI } & \multicolumn{2}{c|}{ CAS } & KT & NKT & TA & \multirow{2}{*}{ C } \\
\cline { 2 - 16 }$n$ & 0.1 & 0.5 & 0.1 & 0.5 & 0.1 & 0.5 & 0.1 & 0.5 & 0.5 & 0.5 & \\
\hline 8744 & L & L & 18 & 16 & L & 5 & 14 & 7 & + & + & 10 & + \\
\hline 8740 & 15 & + & 18 & 17 & 15 & $8-9$ & 7 & + & + & + & 8 & + \\
\hline 8756 & 10 & 9 & L & L & L & 15 & 15 & + & + & + & 10 & + \\
\hline 8656 & 12 & 12 & L & L & L & 15 & 15 & 10 & 5 & + & 10 & + \\
\hline
\end{tabular}

Source: Researched in this paper.

During the experiments with various opportunistic pathogenic strains of Pseudomonas and Stenotrophomonas genera it was found out that the minimal inhibition concentration of researched compounds varies from $0,05 \mathrm{M}$ to $0,01 \mathrm{M}$, depends to different strains, among which there were indicated as non-plasmid strains as well as plasmid-containing strains. In case of researched 4 strains of Pseudomonas syringae it was defined as $0,05 \mathrm{M}$ with inhibition zones diameter $3-5 \mathrm{~mm}$.

As it is shown on table 1, the synthetic derivatives are more effective than the tartaric acid and the natural salts of it. Only for one of strains benzyl mono amino salt is not effective. Imides are more effective and led to lytic effect. According to previous research on other representatives of Pseudomonas, these compounds showed the high efficiency but they are les degradable by soil bacteria then mono amino salts. The usage of tartaric acid derivatives has a range of advantages in comparison with other methods. Due to the previous research, it was detected the absence or resistance spread by plasmids from strains of $P$. aeruginosa, $P$. fluorescens, $P$. putida and $S$. maltophilia which were non sensitive to tartaric acid benzylimide and cyclohexylimide as well as benzyl and cyclohexyl complex salts to different representatives of Pseudomonas, Stenotrophomonas, E. coli and other species of Gramnegative microorganisms (Mikaelyan, et al., 2019).

It is also known that tartaric acid and its derivatives do not inhibit the viability of the probiotic strains of Bifidobacterium and Lactobacillus in gastrointestinal tracts. Examples of amino derivatives: mono-/di-amides and polyamides, was described that exhibit high bactericidal on a number of pathogenic strains (E. coli, P. aeruginosa, Shigella sp., Salmonella typhae, Bacillus subtilis, Bacillus cereus) and fungicidal activity (Trichophytum rubrum) (ElRefaie et al., 2006; Katarzyna et al., 2015; Babayan, 2019). Thus, available natural tartaric acid and the derivatives of it, within the scope of the problem, may be the best alternative solution in case of antibiotic resistant pests and pathogens. 


\section{$2^{\text {nd }}$ International Conference on Advanced Research in SCIENCE, ENGINEERING \& TECHNOLOGY \\ 26-28 March, 2021 \\ FRANCE,PARIS}

\section{Conclusion}

During all the experiments with tartaric acid synthetic derivatives in forms of imides and complex salts of it on phytopathogenic Pseudomonas syringae some strains, the antimicrobial effect of researched 4 compounds was noted. Benzylimide, Cyclohexylimide, as well as benzyl and cyclohexyl mono amino salts had demonstrated themselves as effective antibacterial compounds against the antibiotic resistant strains isolated from soil.

Antimicrobial activity of newly synthesized compounds in a majority of cases was stronger than in case tartaric acid and the well-known derivatives usage. For all the researched strains during the one week of cultivation there were no detected secondary growth after the effect of 4 new synthetic derivatives of tartaric acid. In case of cyclohexyl- substituent containing substances, the effect is stronger than in case of benzyl- substituted derivatives of tartaric acid.

Thus, all the tested semisynthetic substances are recommended for further research of their efficiency as prospective antibacterial agents and ecological safeness, being a potentially biodegradative compounds.

\section{Acknowledgment}

This paper is an output of the scientific project, which was supported by PMI Science \& EIF PhD Support Program.

\section{References}

[1] Nunes C., Duarte A. and Caixeirinho D. (2012). "Organic Acids Concentration in Citrus Juice from Conventional versus Organic Farming”, Acta Hort. (ISHS) vol. 933, pp. 601606.

[2] Cholet C., Claverol S., Claisse O., Rabot A., Osowsky A., Dumot V., Ferrari G., Gény L. (2016). "Tartaric acid pathways in Vitis vinifera L. (cv. Ugni blanc): a comparative study of two vintages with contrasted climatic conditions", BMC Plant Biol. vol. 16 pp. 144.

[3] DeBolt S., Cook D.R. and Ford C.M., (2006).'L-Tartaric acid synthesis from vitamin C in higher plants", Proc Natl Acad Sci U S A., vol.103(14) pp. 5608-5613.

[4] Maroun R.G., Rajha H.N., Vorobiev E. and Louka N. (2017). 7 - Emerging Technologies for the Recovery of Valuable Compounds From Grape Processing ByProducts, Handbook of Grape Processing By-Products Sustainable Solutions, pp. 155-181.

[5] Khan Sh.W., Naz S., Zaidi J.H., Ambreen N., Khan K.M., Perveen Sh. and Miana GH.A. (2012). "Synthesis and Antimicrobial Activity of Chiral Imides from Diacetyl-LTartaric Acid Anhydride and Different Amino Acids", Journal of Pharmacy Research, vol. 5(1), pp. 646-650,

[6] Malik M., Khan Sh. W., Arfan M., Zaidi J. H., Bano A. and Ullah F. (2013). "Biological Evaluation of Chiral Amides Synthesized from Diacetyl-L-tartaric Acid and Aromatic Amines", Asian Journal of Chemistry vol. 25(2) pp. 745-748. 


\section{$2^{\text {nd }}$ International Conference on Advanced Research in SCIENCE, ENGINEERING \& TECHNOLOGY \\ 26-28 March, 2021 \\ FRANCE,PARIS}

[7] Chakhtoura N.G.E. (2018). Therapies for multidrug resistant and extensively drugresistant non-fermenting gram-negative bacteria causing nosocomial infections: a perilous journey toward "molecularly targeted" therapy, Expert Rev Anti Infect Ther., vol.16(2), pp. 89-110.

[8] Adesoji A.T., Ogunjobi A.A., Olatoye I.O. (2015). "Molecular characterization of selected multidrug resistant Pseudomonas from water distribution systems in southwestern Nigeria”, Ann Clin Microbiol Antimicrob., vol.14, pp. 39.

[9] Gomila A., Carratalà J., Eliakim-Raz N., Shaw E., Wiegand I., Vallejo-Torres L., Gorostiza A., Vigo J.M., Morris S., Stoddart M., Grier S., Vank C., Cuperus N., Van den Heuvel L., Vuong C., MacGowan, A., Leibovici L., Addy I. and Pujol M. (2018). "Risk factors and prognosis of complicated urinary tract infections caused by Pseudomonas aeruginosa in hospitalized patients: a retrospective multicenter cohort study", Infect Drug Resist. Vol. 11, pp. 2571-2581.

[10] Jeong, R.-D., Chu, E.-H., Lee, G.W., Park, J.M., Park, H.-J. (2016). "Effect of gamma irradiation on Pseudomonas syringae pv. tomato DC3000 - short communication", Plant Protection Science. vol. 52 (2), pp. 107-112.

[11] Scholz-Schroeder B. K., Soule J.D. and Gross D.C. (2003). "The sypA, sypB, and sypC Synthetase Genes Encode Twenty-Two Modules Involved in the Nonribosomal Peptide Synthesis of Syringopeptin by Pseudomonas syringae pv. syringae B301D". Molecular Plant-Microbe Interactions, vol. 16 (4), pp. 271-280.

[12] De Araujo G.G., Rodrigues F., Gonçalves F.L.T. and Galante D. (2019). "Survival and ice nucleation activity of Pseudomonas syringae strains exposed to simulated high-altitude atmospheric conditions", Scientific Reports, vol. 9:7768, pp. 1 -11

[13] Martin G.B. (2011). "Suppression and Activation of the Plant Immune System by Pseudomonas syringae Effectors AvrPto and AvrPtoB". Effectors in Plant-Microbe Interactions. pp. 123-154.

[14] Sundin G.W., and Wang N.,(2018). Antibiotic Resistance in Plant-Pathogenic Bacteria, Annu Rev Phytopathol, vol. 25;56, pp. 161-180.

[15] WHO: "Critically Important Antimicrobials for Human Medicine", AGISAR,5th rev. ISBN 978-92-4-151222-0, 2017, pp. 20-37.

[16] WHO: "Ten Threats to global health in 2019" (https://www.who.int/emergencies/tenthreats-to-global-health-in-2019).

[17] Dashchyan N.A., Asatryan N.L., Galstyan G.F., Mikaelyan A.R. (2014). “Obtaining Bioactive Additives of Cyclic Structure on the Basis of Optically Active Tartaric Acid", Bulletin of NPUA, Collection of scientific papers, vol. 2, pp. 682-683.

[18] Babayan B. (2019). "The Plasmid Differences In Multi-Drug Resistant Opportunistic Pathogenic Soil Strains of Pseudomonas and Stenotrophomonas," European Journal of Medicine and Natural Sciences (EJMN), V3, No 1, pp. 23-28.

[19] Babayan B.G., Hovhannisyan N.A., Hovhannisyan A.M., Sargsyan A.S., Davidyan T.S., Resistance To $\beta$-Lactam Antibiotics In Some Soil Stenotrophomonas and Pseudomonas, The Scientific-Heritage, Hungary, 2019/05, vol. 2, No.34-2019, pp. 32-38. 


\section{$2^{\text {nd }}$ International Conference on Advanced Research in SCIENCE, ENGINEERING \& TECHNOLOGY \\ 26-28 March, 2021 \\ FRANCE,PARIS}

[20] Birger M.O. (1982) Handbook of microbiological and virological methods of research, M., pp.303-310, 354.

[21] Chaudhary K.D., Khulan A. and Kim J. (2019)."Development of a novel cultivation technique for uncultured soil bacteria", Scientific Reports, vol.9 (6666), pp. 1011.

[22] Babayan B.G., Mikaelyan A.R., Shahinyan S.M. and Bagdasaryan S.A. (2019). "Tartaric Acid New Derivatives Effect Against The Soil Pseudomonas And Stenotrophomonas As A Model For Research of Cave Infection Bacteria Antibiotic Resistance Combating, The book of Abstracts of "International Conference "Caves As Natural \& Cultural Monuments" (Dedicated To The 35th Anniversary of The Armenian Speleological Center)", Yerevan, RA, pp.22 -23.

[23] Mikaelyan A.R., Asatryan N.L., Bagdasaryan S.A. and Babayan B.G. (2019). "Antimicrobial Activity of Newly Synthesized Derivatives of Tartaric Acid Against the Multidrug Resistant Soil Strains of Pseudomonas and Stenotrophomonas", ARICBE/ARICPAS-2019 CAMBRIDGE UNIVERSITY,ABSTRACT BOOK, Cambridge, UK, pp. 1-2.

[24] Katarzyna S., Renata B., Janusz K. and Kamila K. (2015). The Effect of Tartaric Acidmodified Enzyme-resistant Dextrin from Potato Starch on Growth and Metabolism of Intestinal Bacteria. J Plant Pathol Microb vol.6, pp. 269. doi:10.4172/2157-7471.1000269

[25] Babayan B. (2019). "The Plasmid Differences In Multi-Drug Resistant Opportunistic Pathogenic Soil Strains of Pseudomonas and Stenotrophomonas.", 4th International Conference on Medicine and Natural Sciences (ICMN IV) Amsterdam, 26-27 April 2019 Proceedings Book, Amsterdam, Netherlands, pp. 5-10.

[26] El-Refaie K., Fouad I. A.-H., L. Shahada, El-Shanshouri A. E.-R. and El-Newehy M.H. (2006). "Biologically Active Polymers. IV. Synthesis and Antimicrobial Activity of Tartaric Acid Polyamides.”, Journal of Applied Polymer Science, vol. 102, pp. 4780-4790. 